\title{
IMPACT RECONSTRUCTION AND LOCALIZATION ON LAMINATED STRUCTURE
}

\author{
Robert Zemčík ${ }^{1}$, Jan Bartošek², Tomáš Kroupa ${ }^{3}$ \\ 1 University of West Bohemia, Faculty of Applied Sciences, Univerzitní 8, 30100 Pilsen, Czech \\ Republic. E-mail: zemcik@kme.zcu.cz \\ 2 University of West Bohemia, Faculty of Applied Sciences, Univerzitní 8, 30100 Pilsen, Czech \\ Republic. E-mail: bartose4@,kme.zcu.cz \\ 3 University of West Bohemia, Faculty of Applied Sciences, Univerzitní 8, 30100 Pilsen, Czech \\ Republic. E-mail: kroupa@,kme.zcu.cz
}

\section{Introduction}

Finite element model of an airfoil segment made of laminated glass fiber composite is created. The model is calibrated using data from experimental tests, namely the modal characteristics and oscillations measured with laser sensors in selected locations. Passive monitoring method is applied for the reconstruction of impact in unknown location [1]. The location and time variation of impact force is obtained using transferbased functions approach. The dedicated overdetermined and ill-posed inverse problem is solved using Tikhonov regularization [2, 3]. The interpolation of transfer-based functions is used to increase the accuracy of the reconstruction.

\section{Inverse problem}

The methodology used in this work is based on the transfer (or transfer-based) function approach [4]. For a linear system, its response $h$ to an input $f$ can be expressed by convolution $h=f^{*} g$ where $g$ is so-called transfer function and it represents the characteristics of the system $[2,5]$. In order to find the location of impact and to reconstruct the time dependence of the impact force, it is necessary to perform two consecutive steps; a) a calibration procedure, i.e., to perform experimental measurements while recording the corresponding input and response, and to calculate the transfer functions for all combinations of impact (calibration) locations and sensors, and b) a reconstruction procedure, i.e. to reconstruct the force in each possible location for measured response for unknown impact and to seek the impact location, for example by minimizing the error of response reconstruction [6].

\section{Experiment}

A propeller blade segment made of laminated glass-fiber textile composite was used throughout the testing (Fig. 1). It consisted of two parts, a beam (or spar) and an airfoil segment (skin), glued together. The airfoil segment was excited using the impact hammer B\&K 8204 in several locations (one at a time). The force was measured using the embedded force sensor. The response was measured with a set of 4 piezoelectric foil transducers (patches) DuraAct P876.SP1 with shunt resistors $(R=1 \mathrm{M} \Omega)$ and a set of 4 laser sensors Micro-Epsilon optoNCDT 2200. All signals were recorded using NI CompactDAQ device with various $\mathrm{I} / \mathrm{O}$ modules with sampling frequency of approximately $10 \mathrm{kHz}$.

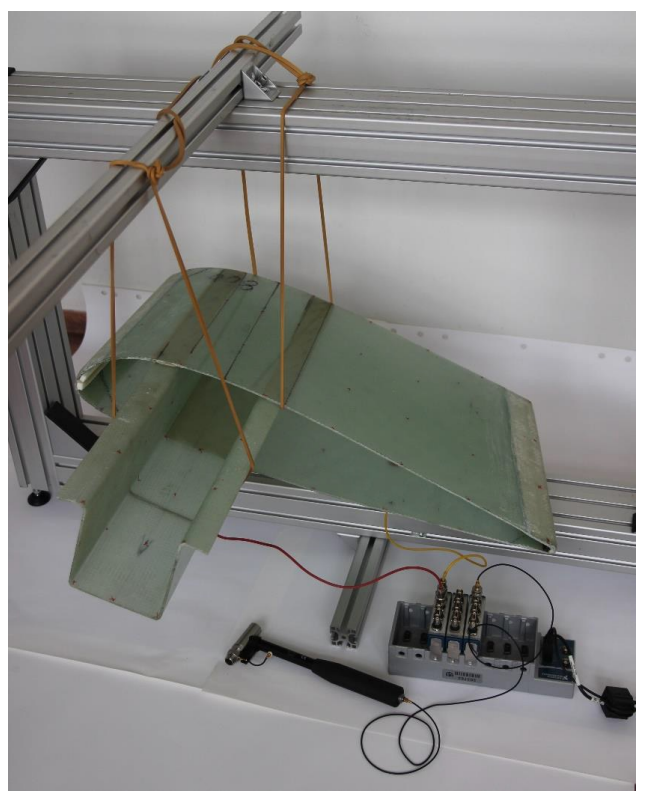

Fig. 1. Experimental setup for free oscillations measurement. 


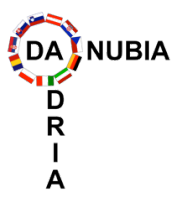

34th Danubia-Adria Symposium on Advances in Experimental Mechanics

University of Trieste, Italy, 2017
First, the blade was hanged on rubber strings and the free oscillations due to the impacts were measured (Fig. 1). This scenario was used for calibration of the numerical model (mainly the elastic properties). Afterwards, the blade was fixed using a turn-table at the beam's end for precise positioning of numerous impacts and the laser beam measurement spots (Fig. 2).

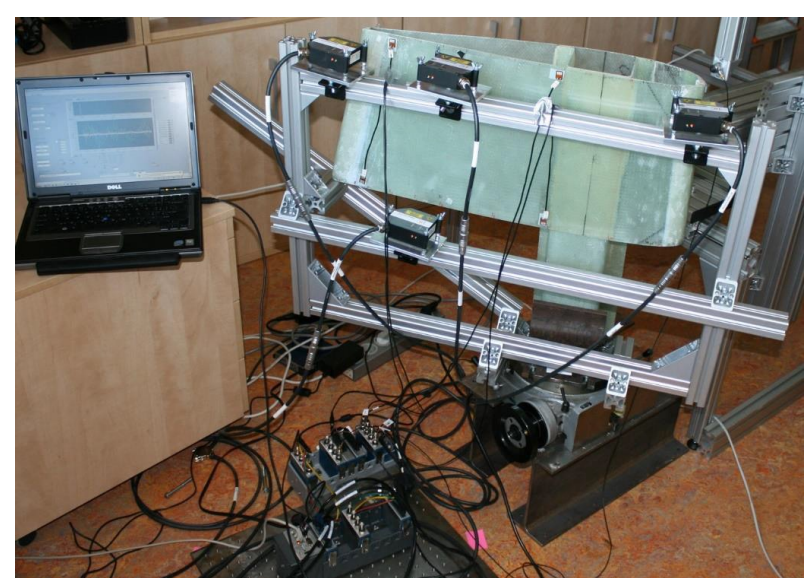

Fig. 2. Experimental setup with turn-table for precise positioning of impacts and sensors.

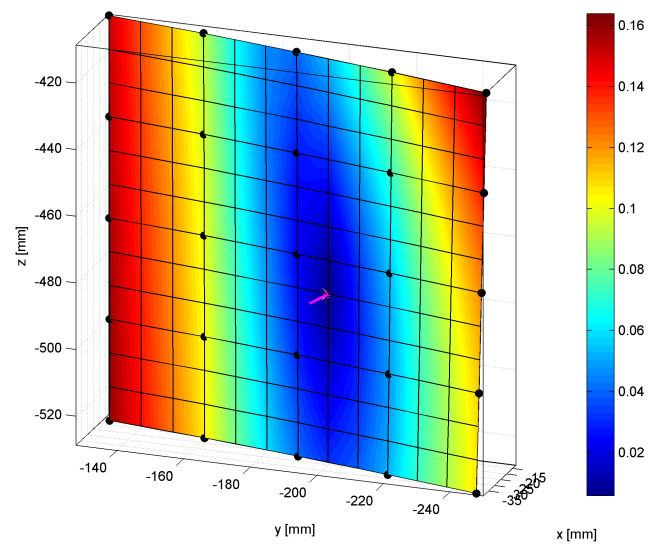

Fig. 3. Localization error contours on $4 \times 4$ grid.

\section{Numerical model and reconstruction}

A numerical model was created for finite element analysis performed in Abaqus solver. The proces used 3D laser scanning probe, CAD tools and, finally, a quadrilateral mesh was created respecting the margins of various regions with different laminated layups (plain- and twill-weave layers were used).

The response of the blade was calculated for 169 location arranged in a $12 \times 12$ grid (covering an area of $120 \mathrm{~mm} \times 120 \mathrm{~mm}$ ) of almost square regions in the central part of the airfoil. The effect of interpolation of transfer functions was then analysed on 5 different simplifications of this grid by reducing the number of hypothetically known impact location by a factor of $2,3,4,6$ and 12 , thus obtaining grids with $12 \times 12,6 \times 6,4 \times 4,3 \times 3$, $2 \times 2$ and $1 \times 1$ regions. An example of impact localization is shown in Fig. 3.

\section{Conclusions}

A methodology for passive structural health monitoring was introduced and applied to laminated structure. The proposed methods for impact location identification and force reconstruction proved to provide results with sufficient accuracy on curved composite structure for selected configurations of calibration data.

\section{Acknowledgements}

This publication was supported by the project LO1506 of the Czech Ministry of Education, Youth and Sports.

\section{References}

[1] Seydel R, Chang FK. (2001) Impact identification of stiffened composite panels: I. System development, II. Implementation studies, Smart Materials and Structures, 10, 354-369 and 370-379.

[2] Gunawan FE, Homma H, Kanto Y. (2006) Two-step B-splines regularization method for solving an ill-posed problem of impact-force reconstruction, Journal of Sound and Vibration, 297, 200-214.

[3] Hu N, Fukunaga H, Matsumoto S, Yan B, Peng XH. (2007) An efficient approach for identifying impact force using embedded piezoelectric sensors, International Journal of Impact Engineering, 34, 1287-1271.

[4] Park J, Chang FK. (2005) System identification method for monitoring impact events, Proc. SPIE, 5758, 189-200.

[5] Jacquelin E, Bennani A, Hamelin P. (2003) Force reconstruction: analysis and regularization of a deconvolution problem, Journal of Sound and Vibration, 265, 81-107.

[6] Atobe S, Kuno S, Hu N, Fukunaga H. (2009) Identification of Impact Force on Stiffened Composite Panels, Transactions of Space Technology Japan, 7, 1-5. 